\title{
Impact of Sleep on Usage of the Smart Phone at the Bedtime- A Case Study
}

\author{
Navya Pratyusha M, Rajyalakshmi K*, Apparao B V, Charankumar G \\ Department of Mathematics, Koneru Lakshmaiah Education Foundation, Vaddeswaram, Guntur-522502, Andhra Pradesh, India
}

Received November 6, 2020; Revised December 17, 2020; Accepted January 20, 2021

\section{Cite This Paper in the following Citation Styles}

(a): [1] Navya Pratyusha M, Rajyalakshmi K, Apparao B V, Charankumar G, "Impact of Sleep on Usage of the Smart Phone at the Bedtime- A Case Study," Mathematics and Statistics, Vol. 9, No. 1, pp. 31-35, 2021. DOI: 10.13189/ms.2021.090105.

(b): Navya Pratyusha M, Rajyalakshmi K, Apparao B V, Charankumar G (2021). Impact of Sleep on Usage of the Smart Phone at the Bedtime-A Case Study. Mathematics and Statistics, 9(1), 31-35. DOI: 10.13189/ms.2021.090105.

Copyright $\mathrm{C} 2021$ by authors, all rights reserved. Authors agree that this article remains permanently open access under the terms of the Creative Commons Attribution License 4.0 International License

\begin{abstract}
Pittsburgh Sleep Quality Index (PSQI) Scoring (Buysse et al. 1989) is a powerful method to measure the sleep quality index based on the scores of various factors namely duration of sleep, sleep disturbance, sleep latency, day dysfunction due to sleepiness, sleep efficiency, need meds to sleep and overall sleep quality. Mainly we focused on the smart phones' usage and its impact on the quality of sleep at the bed time. Many studies have proved that the usage of smart phones at bed time affects the sleep quality, health and productivity. In the present study, we have collected data randomly from the middle-aged adults and observed the relation between gender and the quality of sleep using phi coefficient. It is clearly observed that as we move from males to females, we move negatively from good sleep quality to poor sleep quality. It indicates that males have poor sleep quality than females. We also performed an analysis of variance to test the hypothesis that there is any association between the smart phones' usage and its impact on quality of sleep at bed time.
\end{abstract}

Keywords Sleep Quality, Pittsburgh Sleep Quality Index, Phi Coefficient, Chi Square Test, Kruskal Wallis Test

\section{Introduction}

Smart phones play a key role in our daily life to communicate with each other while away. Enhancement of mobile technology will help us to cater the basic needs and make our lives better. Several apps are providing services to make our dreams come true. The smart phones are useful in many activities such as easily finding where you are and find the routes to go anywhere, easily we can do the official work, spending time wisely reading the news, music, games, entertainment, accessories, food items can be ordered online etc. Smart phone technologies can create the addictive tendencies among the people. This tendency will lead to the major health issues namely skin cancer due to radiation, psychological issue, neck problems, sleep deprivation, eye strain etc., as too much of anything is good for nothing. People spend more time on smart phones during the period of lock down. It leads to the negative impact on health and their sleep quality. Kruskal (1952) introduced a method by using the ranks in one-criterion variance analysis. Buysse et al. (1989) introduced Pittsburgh sleep quality index to measure the quality of sleep. Ahmed et al. (2008) studied prevalence of symptoms and risk of sleep apnea in middle-aged Saudi males in primary care. Liese and Jan (2016) examined the adults' sleep quality when they use smart phone at bed time. Kumar et al (2017a, 2017b) used various statistical techniques to analyze the data. Mohsin and Turki (2019) studied the relation between the sleep quality and sleep hygiene awareness among medical students in King Saud University.

Many investigations have proved that the usage of smart phones at bed time affects the sleep quality, health and productivity. Frequently checking mails, chats, social media are cause to reduce sleep. Doctors suggest that enough quality sleep be guaranteed to prepare ourselves for the next day. Fahdah and Nada (2019) examine the 
prevalence of smartphone usage at bedtime and observed the effect on sleep quality among Saudi non-medical staff working in King Saud University medical city in Riyadh. Following the work of Fahdah and Nada (2019), in the present paper we want to examine the impact of smart phone on the quality of sleep. We observed that the relation between gender and the quality of sleep using phi coefficient. It is clearly observed that as we move from males to females, we move negatively from good sleep quality to poor sleep quality. It indicates that males have poor sleep quality than females. We also performed an analysis of variance to test the hypothesis that there is any association between the smart phones' usage and its impact on quality of sleep at bed time. This cross-sectional study is examined the hypothesis to test the sleep quality using Pittsburgh Sleep Quality Index (PSQI) and the results are presented. Chi-square analysis indicates that the opinions of the persons should not depend on the duration of time spend with mobile at bed time.

\section{Data}

To carry out the analysis, the researcher has collected data from the 121 adults aged between 25 to 62 years during the period of lock down through primary sources. During the lock down period, people spend more time on their mobiles and televisions. So, we have collected data from the adults from April 2020 to June 2020 and observed the effect of smartphone usage on quality of sleep using various statistical techniques.

\section{Methodology}

First, we review the existing literature concerned with the impact of sleep quality and the usage of smart phone before bed time. Different APPs and streaming providers are attracting the customers to use mobiles more frequently. Recent studies have proved that the people are addicted to smart phones and they are spending more time and enhancing their technical knowledge. On the other side of the coin, people are suffering from several health issues, sleeping disorders and psychological issues by spending more time on their smart phones. The following statistical measures have been used to find the relation between the usage of phone at bed time and the sleep quality. Analysis of the results has been obtained using the Software JAMOVI.

\section{Phi Coefficient $(\varnothing)$ :}

If the two variables are dichotomous then the relationship between two variables is called as Phi coefficient $(\varnothing)$. To compute the correlation between gender and quality of sleep, the gender is classified as male and female assigned values 0 and 1 respectively. The sleep of quality can be measured as either good quality (1) and poor quality of sleep (0). It indicates that both the variables are dichotomous variables since the two variables are take value of either 0 or 1 .

$$
\emptyset_{\mathrm{xy}}=\frac{\operatorname{cov}(\mathrm{x}, \mathrm{y})}{\text { standard deviation of } \mathrm{x} \text {. standard deviation of } \mathrm{y}}
$$

Based on the value of phi coefficient we can conclude that the relationship between two variables. The significance can be tested by using the chi-square distribution. The multiplication of $\mathrm{n}$ and $\emptyset^{2}$ is the value of chi-square.

Kruskal Wallis test: Kruskal Wallis test is a non-parametric test and which is used to test the homogeneity of several means. Simply we also treat it as a One-way ANOVA on ranks. Since it is a non-parametric test it doesn't hold the property of normality. Statement of hypothesis is based on medians.

$\mathrm{H}_{0}$ : The medians of all groups are equal

$\mathrm{H}_{1}$ : At least one population median of one group is different from others.

Test statistic

$$
H=(M-1) \frac{\sum_{i=1}^{k} n_{i}\left(\bar{R}_{i .}-\bar{R}\right)^{2}}{\sum_{i=1}^{k} \sum_{j=1}^{n_{i}}\left(R_{i j}-\bar{R}\right)^{2}}
$$

$n_{i}$ representing the number of observations in the group i

$\mathrm{R}_{\mathrm{ij}}$ is the rank of observation $\mathrm{j}$ from group $\mathrm{i}$

$\mathrm{N}$-Total number of observations across all groups

$\bar{R}_{i .}$. Representing the average rank of all observations

$\bar{R}$ average of all the rank of observation $\mathrm{j}$ from group $\mathrm{i}$.

If tie occurs in the data then we correct the above formula and it makes a little difference in the value of $\mathrm{H}$.

Based on the value of $\mathrm{H}$, the decision to accept the null hypothesis or reject can be made. Further we made an analysis chi square to know the opinions of the persons based on the time they spend with mobile at bed time. To analyze the data, we used the software JASP.

\section{Chi-Square test}

The Chi-square $\left(\chi^{2}\right)$ test measures the alignment between two sets of frequency measures. It performs two types of functions namely

i). Goodness of fit: A common use is to assess whether a measure/ observed set of measures follows an expected pattern. The expected frequency may be determined from prior knowledge or by calculation of an average from the given data.

The null hypothesis, $\mathrm{H}_{0}$ is that the two sets of measures are not significantly different.

ii). Measures of Independence: The chi-square test can be used in the reverse manner to goodness of fit. If the 
two sets of measures are compared, then just as you can show they align, you can also determine if they do not align.

The null hypothesis here is that the two sets of measures are similar.

The experimental hypothesis evaluated with the chi-square goodness of fit test is whether or not there is a difference between the observed frequencies of the $\mathrm{k}$ cells and their expected frequencies. The expected frequency of a cell is determined through the use of probability theory or is based on some pre existing empirical information about the variable under study. If the result of the chi-square goodness of fit test is significant, the researcher can conclude that in the underlying population represented by the sample there is a high likelihood that the observed frequency for at least one of the $\mathrm{k}$ cells is not equal to the expected frequency of the cell.

Symbolically,

$$
\chi^{2}=\frac{\left(o_{i}-e_{i}\right)^{2}}{e_{i}} \sim \chi_{(n-1)}^{2} \text { d.f. }
$$

Chi-square test is performed which measures the discrepancy between the observed frequencies and theoretically determined frequencies from the assumed distribution of the same event.

Conditions for the validity of the $\chi^{2}$ - test: $\chi^{2}$ - test is an approximate test for large values of $n$. For the validity of chi-square test of 'goodness of fit' between theory and experiment, the following conditions must be satisfied:

i). The sample observations should be independent

ii). Constraints on the cell frequencies, if any, should be linear.

iii). N, the total frequency should be reasonably large, say greater than 50 .

iv). No theoretical cell frequency should be less than 5 .

- $\chi^{2}$-test depends only one the set of observed frequencies and expected frequencies and on degrees of freedom. It does not involve any population parameters; it is termed as statistic and the test is known as the Non-parametric test or Distribution free test.

Pittsburgh Sleep Quality Index (Buysse et al. 1989): The Pittsburgh Sleep Quality Index (PSQI) is an effective instrument used to measure the quality and patterns of sleep-in adults. It differentiates "poor" from "good" sleep quality by measuring seven components such as subjective sleep quality (SLPQUAL), sleep latency (LATEN), sleep duration (DURAT), habitual sleep efficiency (HSE), sleep disturbances (DISTB), use of sleeping medications (MEDS) and day time dys- function (DAYDYS) over the last month.
Pittsburgh Sleep Quality Index (PSQI) Scoring (Buysse et al. 1989) and scores reportable in publications are as follows:

$$
\begin{gathered}
\text { PSQITOTAL = DURAT + DISTB + LATEN + } \\
\text { DAYDYS + HSE + SLPQUAL + MEDS }
\end{gathered}
$$

Minimum Score $=0$ (better); Maximum Score $=21$ (worse)

Interpretation: TOTAL $\leq 5$ associated with good sleep quality

TOTAL $>5$ associated with poor sleep quality

All the seven components have considered the minimum score (0) is better and maximum score (3) is worse.

\section{Results and Discussion}

A sample of 121adults both men and women have displayed a Standard deviation of 3.04. They got a minimum value of 2 , maximum value of $14 \&$ average value (mean) of 7.6.

Table 1. Gender and Quality of sleep based on PSQI

\begin{tabular}{|c|c|c|c|c|}
\hline Variable & Category & $\mathrm{N}$ & Mean & S.D. \\
\hline \multirow{2}{*}{ Gender } & Male & 64 & 8.125 & 2.909 \\
\cline { 2 - 5 } & Female & 57 & 7.018 & 3.102 \\
\hline
\end{tabular}

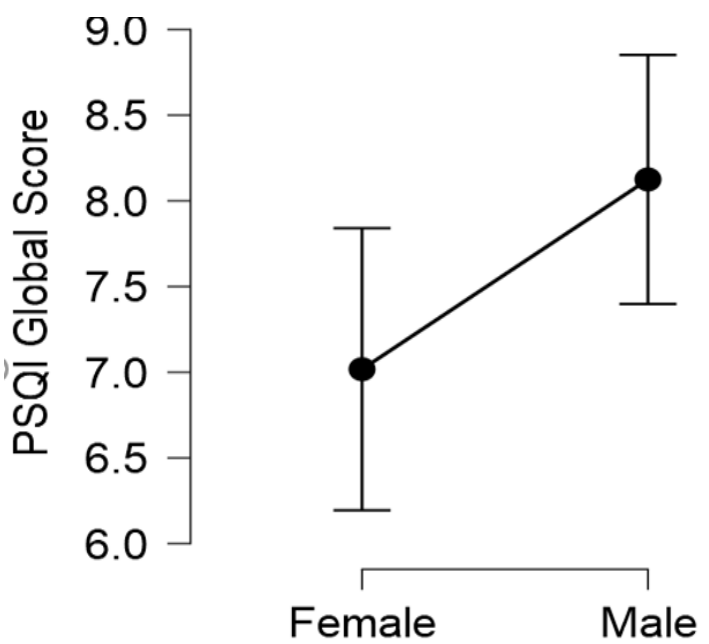

The relation between gender and the quality of sleep using phi coefficient we got the value of -0.10996 . The value of phi coefficient is indicating that as we move from males to females, we move negatively from good sleep quality to poor sleep quality. It indicates that males have poor sleep quality than females. We also performed an analysis of variance to test the hypothesis that there is any association between the smart phones' usage and its impact on quality of sleep at bed time. 
Table 2. Kruskal-Wallis Test

\begin{tabular}{|c|c|c|c|}
\hline Factor & Statistic & Degrees of freedom & $\mathrm{p}$ \\
\hline PSQI Duration & 30.06 & 3 & $1.341 \mathrm{e}-6$ \\
\hline PSQIDIST & 42.97 & 3 & $1.876 \mathrm{e}-7$ \\
\hline PSQI LATEN & 25.58 & 3 & $1.167 \mathrm{e}-5$ \\
\hline PSQI Sleep Quality & 43.78 & 3 & $1.684 \mathrm{e}-9$ \\
\hline PSQI DaysDYS & 23.26 & 3 & $3.560 \mathrm{e}-5$ \\
\hline PSQIHSE & 48.36 & 3 & $1.781 \mathrm{e}-10$ \\
\hline PSQI MEDS & 3.68 & 3 & 0.298 \\
\hline
\end{tabular}

From the results of table- 2 we observed that seven factors are influenced by the usage of mobile at bed time and it leads to affecting the sleep quality. Kruskal-Wallis test is useful to determine the variation among the groups on ranks.

Table 3. Summary statistics of various factors influencing the sleep quality

\begin{tabular}{|c|c|c|c|c|c|c|c|c|c|c|c|c|}
\hline & \multicolumn{4}{|c|}{$\mathrm{N}=121$} & \multicolumn{4}{c|}{ Median } & \multicolumn{5}{c|}{ Z } \\
\hline & 0 & 1 & 2 & 3 & 0 & 1 & 2 & 3 & 0 & 1 & 2 & 3 \\
\hline PSQI Duration & 42 & 38 & 27 & 14 & 4 & 8 & 9 & 9 & -5.3 & 1.98 & 1.99 & 2.5 \\
\hline PSQIDIST & 30 & 44 & 23 & 24 & 7 & 5 & 9 & 10 & -0.43 & -5.67 & 3.81 & 3.56 \\
\hline PSQI LATEN & 29 & 40 & 26 & 26 & 6 & 6 & 9 & 9 & -2.60 & -2.92 & 2.76 & 3.29 \\
\hline PSQI Sleep Quality & 54 & 24 & 19 & 24 & 6 & 8 & 10 & 10.5 & -5.75 & -0.07 & 2.49 & 4.97 \\
\hline PSQI DaysDYS & 19 & 47 & 37 & 18 & 7 & 6 & 8 & 10 & -0.81 & -3.76 & 1.76 & 3.70 \\
\hline PSQIHSE & 44 & 27 & 31 & 19 & 5 & 8 & 9 & 10 & -6.51 & 0.78 & 3.16 & 3.92 \\
\hline PSQI MEDS & 106 & 8 & 6 & 1 & 8 & 4 & 8.5 & 8 & 0.52 & -1.64 & 1.07 & 0.06 \\
\hline
\end{tabular}

Table 4. Time spent on smart phone before sleeping

\begin{tabular}{|c|c|c|c|c|c|}
\hline \multirow{2}{*}{$\begin{array}{c}\text { Time spent spend on smart } \\
\text { phone before sleeping } \\
\text { (minutes) }\end{array}$} & \multicolumn{5}{|c|}{ Excessive Smartphone Usage before bedtime affects sleep quality } \\
\cline { 2 - 6 } & $\begin{array}{c}\text { Strongly } \\
\text { disagree }\end{array}$ & Disagree & Neutral & Agree & Strongly agree \\
\hline $0-15$ & 0 & 2 & 8 & 14 & 7 \\
\hline $16-30$ & 1 & 4 & 3 & 20 & 4 \\
\hline $31-45$ & 1 & 1 & 8 & 11 & 2 \\
\hline $46-60$ & 0 & 1 & 3 & 10 & 3 \\
\hline$>60$ & 1 & 2 & 2 & 7 & 6 \\
\hline
\end{tabular}

From the above table, we get the chi-square value $=15.54$, chi-square table value $=26.29$. Based on the values of calculated and table we can conclude that the opinion of the persons are independent on the excessive smart phone usage before bed time affects sleep quality.

Table 5. Age wise impact on sleep quality

\begin{tabular}{|c|c|c|c|}
\hline \multirow{2}{*}{ Age } & \multicolumn{2}{|c|}{ Total number of responses with respect to the Quality of sleep } \\
\cline { 2 - 4 } & Good & Poor & Total \\
\hline Below 40 & 12 & 24 & 56 \\
\hline Between 40 and 50 & 8 & 44 & 33 \\
\hline Above 50 & 11 & 22 & 121 \\
\hline Total & 31 & 90 & 52 \\
\hline
\end{tabular}

From the above table, we get the chi-square value $=5.0177$, chi-square table value $=5.99$. Based on the values of calculated and table we can conclude that the quality of sleep should not depend on their age. Smart phone usage before bed time is affecting all age people equally. 


\section{Conclusions}

In the present study, we focused on the analysis of data to observe cross sectional study of different factors and its impact on sleep quality when we use the mobile phone at bed time. We have collected data through primary source and analyze the data using Descriptive statistics, phi coefficient, Kruskal Wallis test and Pittsburgh sleep quality index. The value of phi coefficient is indicating that as we move from males to females, we move negatively from good sleep quality to poor sleep quality. It indicates that males have poor sleep quality than females. An analysis of variance to test the hypothesis that there is any association between the smart phones' usage and its impact on quality of sleep at bed time for different factors can be studied through Kruskal Wallis test. Results of table (2) indicating that the sleep quality (SLPQUAL), sleep latency (LATEN), sleep duration (DURAT), habitual sleep efficiency (HSE), sleep disturbances (DISTB) shows significance at 0.05 level of significance whereas the factor sleeping medications (MEDS) is not significant at the same level of significance. Table (3) gives us an idea about the ' 7 ' factors median and the number of observations at ' 0 ' (better), ' 1 ' (Average), ' 2 ' (Poor), and ' 3 ' (Worse) along with the $\mathrm{z}$ values. Table (5) gives an idea about the quality of sleep based on their age.

\section{REFERENCES}

[1] Ahmed S BaHammam, Mohammed S Alrajeh, Hamdan H Al-Jahdali, Abdulaziz A BinSaeed (2008), Prevalence of symptoms and risk of sleep apnea in middle-aged Saudi males in primary care, Saudi Med J, 29(3), 423-426.

[2] Bodiga, V L; Eda, SR; Bodiga, S (2014). Advanced glycation end products: role in pathology of diabetic cardiomyopathy, HEART FAILURE REVIEWS, 10.1007/s10741-013-9374-y.

[3] Buysse DJ, Reynolds CF, Monk TH, Berman SR, Kupfer DJ (1989). The Pittsburgh sleep quality index: A new instrument for psychiatric practice and research. Psychiatry Res. 28:193-213.

[4] Chaitanya G.K., Soundarya U.L., Chandan M., Sandeep S. (2017).Health care monitoring using wifi through mobile devices, Journal of Advanced Research in Dynamical and Control Systems, 9(14), 608-617.

[5] Charankumar, G and Rajyalakshmi K (2019). Measuring Spatial Dependencies of Various Spatial Objects Related To the Road Safety Discrepancies, International Journal of
Innovative Technology and Exploring Engineering, 8(11), 2278-3075.

[6] Fahdah A Alshobaili and Nada A AIYousefi (2019). The effect of smartphone usage at bedtime on sleep quality among Saudi non- medical staff at King Saud University Medical City, J Family Med Prim Care, 8(6), 1953-1957.

[7] Jayanthi N., Babu B.V., Rao N.S. (2017). Survey on clinical prediction models for diabetes prediction, Journal of Big Data, 4 (1), 26-30.

[8] JASP Team (2020). JASP (Version 0.14).

[9] Kishore, PVV; Sastry, ASCS; Rahman, MZU (2016), Double Technique for Improving Ultrasound Medical Images, JOURNAL OF MEDICAL IMAGING AND HEALTH INFORMATICS.

[10] Kruskal; Wallis (1952). "Use of ranks in one-criterion variance analysis". Journal of the American Statistical association, 47 (260): 583-621.

[11] Kumar D.P., Rajyalakshmi K., Asadi S.S. (2017a). A model analysis for the promotional techniques of cell phone subscriber identity module (SIM) cards, International Journal of Civil Engineering and Technology, 8 (9), 889-897.

[12] Kumar D.P., Rajyalakshmi K., Asadi S.S. (2017b). Analysis of mobile technology switching behavior of consumer using chi-square technique: A model study from Hyderabad, International Journal of Civil Engineering and Technology, 8 (9), 99-109.

[13] Liese Exelmans and Jan Van den Bulck (2016). Bedtime mobile phone use and sleep in adults, Soc Sci Med, 148, 93-101.

[14] Mohsin Alshahrani and Yousef Al Turki (2019). Sleep hygiene awareness: Its relation to sleep quality among medical students in King Saud University, Riyadh, Saudi Arabia, J Family Med Prim Care, 28, 8(8), 2628-2632.

[15] Murty A.S.R., Satyanarayana M., Devi Vara Prasad I (2018). Compressor health monitoring using IOT, International Journal of Mechanical and Production Engineering Research and Development, 8(3), 117-124.

[16] Phani Babu K., Siva Nageswara Rao G (2019). Smart healthcare system, International Journal of Innovative Technology and Exploring Engineering, 8 (12), 4184-4188.

[17] Randive, SN; Rahulkar, AD; Senapati, RK, LVP (2018). Extraction and triplet-based segmentation for diabetic retinopathy recognition, EVOLUTIONARY INTELLIGEN CE.

[18] Razia S., Narasingarao M.R. (2017). A neuro computing frame work for thyroid disease diagnosis using machine learning techniques, Journal of Theoretical and Applied Information Technology, 95(9), 1996-2005. 